\title{
An investigation into the peri-operative nutritional management of open colorectal surgery patients in major Australian hospitals: A comparison with the ERAS guidelines.
}

\author{
Katie Wykes ${ }^{1}$, Karen McInnes ${ }^{2}$, Shelley Wilkinson ${ }^{3,4}$ \\ ${ }^{1}$ Student Dietitian, Griffith University, Gold Coast, Queensland, 4222. \\ 2 Dietitian, Mater Health Services, South Brisbane, Queensland, 4101. \\ ${ }^{3}$ Research Dietitian, Mater Medical Research Institute, Mater Health Services, South \\ Brisbane, Queensland, 4101 \\ ${ }^{4}$ Research Dietitian, Research Centre for Clinical and Community Practice Innovation, \\ Griffith University, Gold Coast, Queensland, 4222.
}

Extensive research has produced the Enhanced Recovery After Surgery (ERAS) guidelines for best practice peri-operative management of colorectal surgery patients, including their nutritional management. Despite clear guidelines with demonstrated improved patient outcomes, further research shows poor implementation of ERAS nutrition guidelines (NGs). This study investigated nutritional management of colorectal surgery patients in Australian tertiary hospitals and compared current procedures with the ERAS NGs. We also evaluated the success of and identified barriers to ERAS NGs implementation in major Australian Hospitals. Dietitians from ten Australian hospitals completed telephone interviews regarding local dietetic practices and perceived barriers and enablers to implementing ERAS NGs. Thematic analysis was used to expose common themes. Results show that, despite wide awareness of the ERAS NGs, all dietitians have been unable to fully implement ERAS based NGs within their hospitals. Only three hospitals have made significant changes in patient care protocols to ensure evidence-based practice. The most consistently implemented guideline was the early removal of nasogastric tubes; NGs with most difficulty in implementing were pre-operative carbohydrate supplementation and not using bowel 'prep'. Analysis of implementation 'issues' has resulted in 13 recommendations to improve ERAS NG adoption. These recommendations include: presenting a rationale as to why change is needed, promoting the importance of nutrition, engaging a multidisciplinary team, promoting the protocol to clinical staff, recruiting champions, promoting the preservation of clinical judgement, creating timelines, displaying confidence, selecting appropriate times to initiate practice changes, learning from other facilities, educating staff, displaying a coordinated team approach and actively engaging patients.

Contact author: Katie Wykes - katiewykes@gmail.com

No funding received 